\title{
Force Control of Piezoelectric Walker
}

\author{
Tarik Uzunovic \\ Faculty of Electrical Engineering \\ University of Sarajevo \\ Sarajevo, Bosnia and Herzegovina \\ Email: tuzunovic@etf.unsa.ba
}

\author{
Edin Golubovic \\ Inovatink Engineering \\ Istanbul, Turkey \\ Email: edin@inovatink.com
}

\author{
Asif Sabanovic \\ Faculty of Engineering and Natural Sciences \\ Sabanci University \\ Istanbul, Turkey \\ Email: asif@sabanciuniv.edu
}

\begin{abstract}
This paper is concerned with the force control of a walking piezoelectric motor, a commercially available Piezo LEGS motor. The motor is capable of providing high precision positioning control on nanometer scale, but also relatively high forces up to $6 \mathrm{~N}$. The proposed force control algorithm is very simple, but effective, and it is based on a recently presented coordinate transformation. The transformation allows definition of the driving waveforms for the motor according to a desired motion of the motor legs in the plane of motion. Such a possibility opens a path for creating the $y$-direction interaction force between the motor legs and the rod which is enough to ensure no relative motion between the legs and the rod. Once that is achieved, one can control the $x$-direction force imposed by the motor rod on its environment. The presented force control scheme has been successfully validated through a series of experiments.
\end{abstract}

\section{INTRODUCTION}

One of the most important trends in modern industry is miniaturization of products. Such a situation naturally requires very small production devices which can exhibit high precision positioning with effectively controlled force. Therefore, high precision position and force control are inevitable in the process of development of microtechnology, which further includes micromanufacturing, microassembly and micromanipulation.

A group of actuators which can respond to the stated challenges regarding precision and force control is consisting of piezoelectric (PZT) actuators. They are particularly useful in applications requiring high precision positioning, very fast response, and significant force-to-volume ratio.

One can find numerous examples of piezoelectric actuators' applications in the literature. These applications are discovered in many different fields. Thus, PZT motors were used for the design of the handheld tremor-canceling manipulator [1]. In [2], the authors demonstrated usage of PZT motors in the development of an MR-compatible actuation system built for a parallel force-feedback exoskeleton used for measurement and/or assistance of wrist pointing movements during functional neuroimaging. Additionally, PZT actuation is utilized for the development of a nanomanipulation system for operation inside scanning electron microscopes (SEM) [3]. In [4], a fully actuated 6-DOF robot is presented. The robot is actuated by piezoelectric motors and it is capable of performing both prostate biopsy and brachytherapy procedures with MR image guidance. Piezoelectric actuators are being used as a part of a hybrid $x y$ stage used in a novel large measurement- range atomic force microscopy (AFM) system [5]. In [6], the authors presented mechanical design, optimisation and tracking control of a flexure-based positioning stage capable to generate coupled angular and linear motions. The mechanism employs two piezoelectric actuators, with output rotation and translation proportional to the sum and difference of the input displacements.

Various different modeling and control approaches for PZT actuators and mechanisms based on these actuators were reported in the literature. In particular, a pretty general framework was introduced in [7] concerned with modeling and control design for PZT positioning stages. One can find an extensive overview of modeling and control approaches for nanopositioning stages actuated by PZT actuators in [8]. In [9], a high-speed tracking control approach for third-order piezo-actuated nanopositioning stages, which extends the vibration control strategies tailored for damping the resonant modes of second-order systems to third-order systems is presented. A controller design for a high precision rotational stage based on a piezoelectric actuator is given in [10]. The proposed controller is actually a 2-degree of freedom (2DOF) controller, adopted and designed in order to ensure good reference tracking and high disturbance rejection due to the external noise. In addition, an approach based on modified repetitive control is proposed for high-speed tracking control of piezo-actuated nanopositioning stages in [11]. A low-order repetitive control design in continuous-time for piezo-based nanopositioning system was proposed in [12]. The authors in [13] introduced a novel modeling and identification for approach for piezoelectric-actuated stages by cascading hysteresis nonlinearity with linear dynamics, which is described as a Hammerstein-like structure.

The motor used in this research is a piezoelectric walker, commercially available Piezo LEGS motor. The motor is described in detail in patents [14], [15], [16]. The motor was used until now in high precision positioning and force control applications. In [17], the motor actuated a single DOF positioning stage. The proposed control system used position feedback strategy. Delay varying repetitive control was introduced in [18] and utilized for positioning control of a stage driven by a walking piezoelectric motor. Adaptive control of the Piezo LEGS motor was in the focus of the authors in [19]. In [20], gain scheduling control of a walking piezo actuator was discussed. A Insect-inspired drive mechanism 


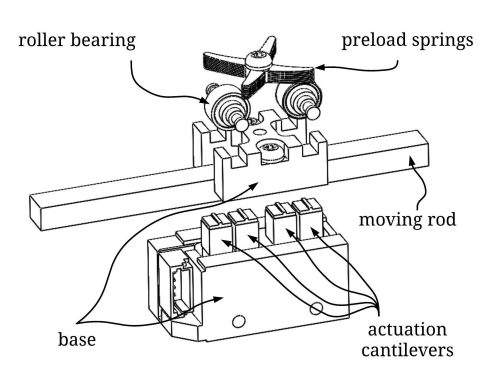

Fig. 1: Piezo LEGS motor.

for the Piezo LEGS motor is introduced in [21], and the authors showed that it truly improves performance of the motor when generating force capabilities and maximal drive velocity are analyzed. Lately, implementation of the FPGA-based and DSC-based control systems for the Piezo LEGS motor were reported in [22] and [23]. The Piezo LEGS motor used in this research was utilized to perform force control in [24] and [25]. The presented control scheme required extensive modeling effort, which was tried to be avoided in this paper.

In this paper, a novel force control algorithm for the Piezo LEGS motor is proposed and it is based on our novel driving strategy for this motor based on a coordinate transformation [26]. The transformation allows independent control of the motor legs in both directions ( $x$ - and $y$-directions) in their plane of motion. Such a possibility allows definition of a simple but effective force control algorithm, as illustrated in this paper.

\section{System Description}

The research in this paper is centered around the exploration of force control capabilities of commercially available Piezo LEGS motor [14], [15], [16]. This motor is capable of very precise positioning and has high force/volume ratio which makes it ideal for precise positioning application where size and weight constraints play dominant role in design [19], [23], [26]. This work additionally demonstrates the force control capabilities of such motor. The assembly of Piezo LEGS motor is shown in Fig. 1. Constituent parts of Piezo LEGS motor assembly are base, actuation cantilevers, moving rod, roller bearings and preload springs.

Base is machined out of steel material and consists of two parts. Bottom part of the base hosts actuation cantilevers and actuation voltage terminals in its structure. Top part of the base hosts roller bearings and preload springs. Bottom and top part of the base are mounted together using screws.

Roller bearings are constructed as classical two-race bearings. Outer race is pressed on the moving rod to allow for its motion with very small rolling resistance and inner race is pieced together with small shaft running through its inner circle. This shaft is thinned at the end and mounts on the top base part. Rolling bearings are constructed out of aluminum.

Preload springs are leaf, " $x$ " shaped pieces of metal, stacked together and held to the top base part with screw running through their center. Each edge of this "x" shaped structure

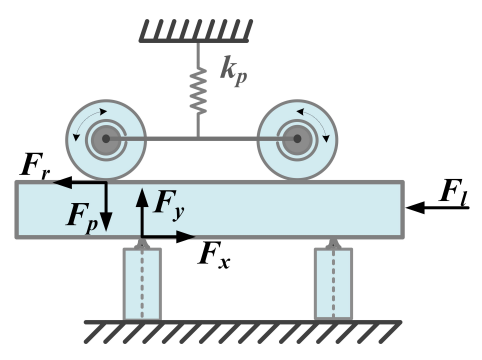

Fig. 2: Free body diagram of the motor.

holds thinned end of the roller bearing shaft. Such construction of preload springs allows for an effective way to hold roller bearings against moving rod while opening a way to set desired preload force (stiffness).

Moving rod is constructed out of ceramic material and resides sandwiched between preload spring pressed roller bearings and tips of the actuation cantilevers. Rod is free to move in the direction of motion and its motion is constrained in other directions by the assembly.

PiezoLEGS motor is actuated by four piezoelectric cantilevers. Cantilevers are constructed as piezoelectric bimorphs with two active layers. Each active layer is constructed from piezo stacks whose direction of stress and polarization are the same, i.e. only nonzero piezoelectric coefficient is $d_{33}$. Such construction of a cantilever enforces the alignment of the direction of induced strain and direction of applied electric field so the cantilever can bend when one active layer contracts and the other one expands under applied voltage. Furthermore, coordinated control of actuation voltages can result is both deflection and elongation, thus planar motion, of the tip of the cantilever. Each cantilever is driven with two voltage sources and the cantilevers are driven in the pair of two, meaning that in total four different voltage signals are required for motor driving. The tips of cantilevers are made out of aluminum base ceramic material that has high stiffness and high friction coefficient. Actuation cantilevers are further denoted simply as "legs".

The driving principle of PizeoLEGS motor is based on the friction formed at the contact point between legs and the rod. When legs are driven using phase shifted operation, one pair is brought into mechanical contact with the moving rod (grip), and then moved in the desired direction (move); afterwards, this pair releases the moving rod (release) and returns to the initial position (return). As soon as the release operation of the first pair of legs occurs, another pair is brought into the grip position. If the grip-move-release-return action is denoted as a step, then the rod is brought into the target position by the repetition of a number of steps.

Figure 2 shows the free-body diagram of the Piezo LEGS motor with a pair of legs. Forces acting on the rod are $F_{r}$, the rolling friction of the roller bearings, $F_{p}$ is the preload force exerted on the rod by equivalent preload spring with coefficient $k_{p}, F_{y}$ is the $y$-direction interaction force between the legs and the rod, $F_{x}$ is the $x$-direction interaction force between the legs 
and the rod, and $F_{l}$ is an externally applied load force in the direction opposite to that of the rods motion.

Shown forces define the interaction of the rod and the legs. In normal operation of the Piezo LEGS motor, there is no relative motion between the legs and the rod and interaction is defined only by stiction force. That situation can be achieved by setting $F_{y}$ to a high value. In this case, the deflection of the legs in $x$-direction will cause the movement of the rod for the same amount. Force $F_{y}$ is generated once the nonzero sum of voltages is applied to the legs. The rod moves in the direction of this force when its magnitude becomes larger than the magnitude of $F_{p}$. The condition for the motion of the rod in x-direction requires the magnitude of $F_{x}$ to exceed the sum of magnitudes of forces $F_{l}$ and $F_{r}$. Additionally, the maximum value of force $F_{x}$ in normal operation is limited by the value of stiction force threshold. The stiction force threshold is determined by the product of magnitude of $F_{y}$ and stiction coefficient $\mu_{s}$. Conditions for the motion of the rod in $\mathrm{x}$ - and $\mathrm{y}$-directions can be summarized as,

$$
\begin{aligned}
& \text { for } \Delta y_{r}>0, F_{y}>F_{p} \\
& \text { for } \Delta x_{r}>0, F_{x}>F_{r}+F_{l} \\
& \max \left(F_{x}\right)=\mu_{s} F_{y}
\end{aligned}
$$

In (1), $\Delta x_{r}$ and $\Delta y_{r}$ denote the change in position of rod in $x$ - and $y$-directions, respectively.

\section{DRIVING PRINCIPLE}

The Piezo LEGS motor used in this research was driven according to the approach discussed in detail in [26]. The approach is based on a coordinate transformation, and it will be briefly presented here for the sake of completeness of this paper.

For the frequency range limited with $20 \mathrm{kHz}$, which is the maximum sampling frequency used in our applications, and achievable with available hardware, one can disregard dynamics of the motor legs, i.e. consider it much faster than these $20 \mathrm{kHz}$. Therefore, static relations can be taken as valid relationship between driving voltages and motion of the legs. Here, it is assumed that motion of the first pair of legs is being controlled by driving voltages $V_{1}$ and $V_{2}$, while $V_{3}$ and $V_{4}$ are driving the second pair. In addition, it is assumed that all legs have the same characteristics. Therefore, the displacement of the legs from one pair is always the same.

The displacements of the legs from each pair can be described, according to the relations given in our previous publications [22], [23], [26], in the following form

$$
\begin{aligned}
& \Delta x_{p 1 / 2}(t)=k_{1}\left[V_{1 / 3}(t)-V_{2 / 4}(t)\right] \\
& \Delta y_{p 1 / 2}(t)=k_{2}\left[V_{1 / 3}(t)+V_{2 / 4}(t)\right] .
\end{aligned}
$$

In (2), the constants $k_{1}$ and $k_{2}$ depend on the mechanical properties of the legs. For each pair, the difference of its driving voltages controls displacement in the $x$-direction. On the other hand, the sum of the voltages is controlling $y$ direction displacement. Therefore, the displacements are coupled in the sense that both driving voltages are controlling both displacements. The driving scheme used in this paper was first introduced to decouple these displacements. Let the vector of driving voltages $\mathbf{V}=\left[\begin{array}{llll}V_{1} & V_{2} & V_{3} & V_{4}\end{array}\right]^{\mathrm{T}}$ be defined as

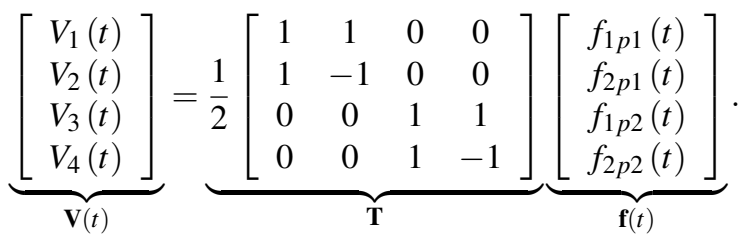

In the last equation, the vector $\mathbf{f}$ will be called the moving vector and its components, $f_{1 p 1}, f_{2 p 1}, f_{1 p 2}, f_{2 p 2}$ are time functions, yet to be defined. The matrix $\mathbf{T}$ is a nonsingular matrix which defines mapping between the moving vector and driving voltages. When (3) is introduced in (2), it can be written

$$
\begin{aligned}
& \Delta x_{p 1 / 2}(t)=k_{1} \cdot f_{2 p 1 / 2}(t) \\
& \Delta y_{p 1 / 2}(t)=k_{2} \cdot f_{1 p 1 / 2}(t) .
\end{aligned}
$$

From (4), one can conclude that displacements of the legs in the $x$ - and $y$-directions can be independently specified if one specifies the moving vector components. The components $f_{1 p 1}$ and $f_{1 p 2}$ specify the $y$-direction displacements. One can use them to control $y$-direction interaction force between the legs and the ceramic rod. This force should be kept high enough to enable normal operation of the motor, i.e., no-slip motion between the pair of legs which is driving and the rod and the rod itself. In contrary, the functions $f_{2 p 1}$ and $f_{2 p 2}$ are defining the $x$-direction motion of the legs. Therefore, they can be used for specification of the $x$-direction motion profile of the rod, and by that used to control $x$-direction force imposed by the rod on its environment. Control of this force is in the focus of the paper. The last equation also shows that for each desired (of course, achievable) trajectory of the legs in the plane of motion, $(x, y)$ plane, one can synthesize driving voltages that will enforce such trajectory.

According to the presented scheme, there is infinite number of possible definitions of the driving vector. When making a definition, one has to satisfy certain constraints which were discussed in detail in [26]. The definition used in this research was selected because of several things. First, their implementation is simple, since they are piecewise linear function, which is desired for fast calculations in real-time implementations. In addition, the used allows two conditions to be satisfied by appropriate selection of the parameters of the moving vector: i) resultant force applied by the legs on the motor rod resides inside the cone of friction, and ii) the rod has constant velocity within one step. The components of the moving vector are all defined as periodic functions with basic period $T$, and they are depicted in Fig. 3. The parameters $b$ and $d$ define amplitudes of the components. On the other hand, the parameter $c_{\text {min }}$ is determined in experiments from the contribution of a pair to the rods motion. In particular, if $f_{1 p 1} \leq c_{\min }\left(f_{1 p 2} \leq c_{\text {min }}\right)$, the first (second) pair's $y$-direction displacement is not big enough to produce $y$-direction interaction force greater than $F_{\text {lim }}$, i.e., the corresponding pair is not influencing the rod's motion. In the description of the force control algorithm it will be discussed how parameters of the moving vector components 


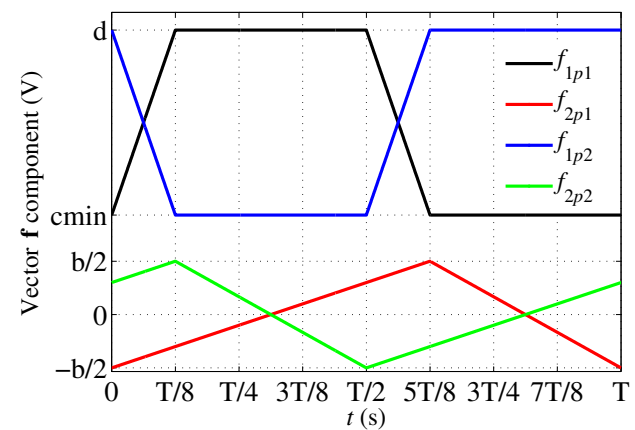

Fig. 3: Time waveforms of the vector $\mathbf{f}$ components.

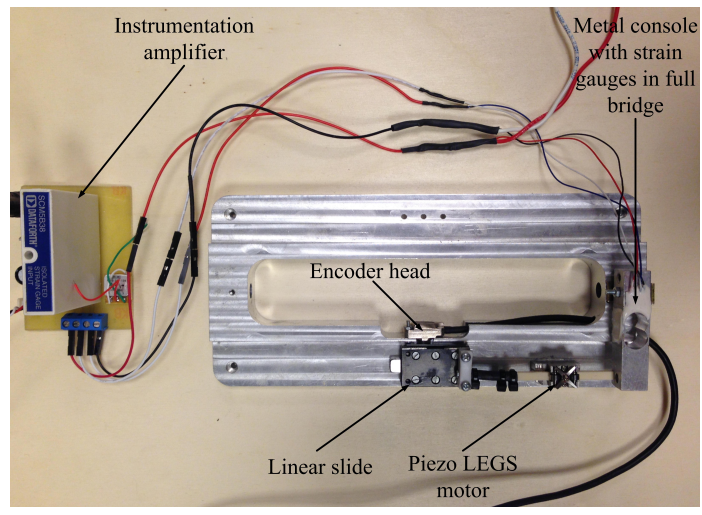

Fig. 4: Experimental setup.

influence the motor operation.

\section{ForCE CONTROL}

\section{A. Force Measurement}

In order to perform force control, an experimental setup was created in which the motor was interacting with a metal console which could be bent, and the goal was to control the interaction force between the motor and console. The interaction force imposed by the rod was measured by four strain gauges mounted to the console in full bridge configuration. It is well known that full bridge configuration gives linear relationship between measured force and output voltage for small relative changes of the resistances of the gauges. Since the measurement console was taken from a kitchen scale, its mechanical construction ensures small deflections of the metal console to which the gauges are mounted. Therefore, the linear relationship is ensured. The output voltage was amplified by an instrumentation amplifier, in order to bring it to volt-range, so it can be acquired by available analog-to-digital converter. The complete experimental setup is shown in Fig. 4.

With the aim to identify characteristics of the created measurement setup, a series of measurements was done, by loading the measurement device with iron weights of known mass. The obtained measurement results enabled us to get characteristic of the device, which gives relation between amplified output voltage and measured force.

\section{B. Control System}

The control algorithm relies on the interaction force modeled as linear spring, which is very often done in force control applications. Therefore, if the interaction force is modeled as

$$
F=k_{e} x
$$

where $k_{e}$ is the spring constant of the environment opposing the motor motion, and $x$ is the motor position, with assumption that interaction force exists only for $x>0$. If the reference force is $F^{r e f}=k_{e} x^{r e f}$, one can express the force tracking error using the position tracking error $e_{p}$ as

$$
e_{F}=F^{r e f}-F=k_{e}\left(x^{r e f}-x\right)=k_{e} e_{p} .
$$

In this way, the force control can be executed using the position control algorithm, that has been already proposed in previous papers [23], [26]. The mentioned position control algorithm was named virtual time control, since it is changing the argument of the moving vector components. Since their argument is actually time, and this control algorithm is increasing or decreasing that in order to decrease the position tracking error, the argument becomes some virtual time. Thus, the name of the algorithm was chosen as virtual time control.

The virtual time control can be described with the following pseudocode.

$$
\begin{aligned}
& \text { : procedure VirtualTimeControl }\left(e_{p}\right) \\
& \text { while TRUE do } \\
& \left|e_{p}\right| \leftarrow \operatorname{abs}\left(e_{p}\right) \\
& f_{\text {old }} \leftarrow f \\
& \text { if }\left|e_{p}\right| \leq e_{\text {down }} \text { then } \\
& f \leftarrow f_{\text {min }} \\
& \text { else if } e_{\text {down }}<\left|e_{p}\right|<e_{u p} \text { then } \\
& f \leftarrow f_{\text {min }}+\left(f_{\text {max }}-f_{\text {min }}\right)\left(\frac{\left|e_{p}\right|-e_{\text {down }}}{e_{\text {up }}-e_{\text {down }}}\right)^{2} \\
& \text { else } \\
& f \leftarrow f_{\max } \\
& \text { end if } \\
& T \leftarrow 1 / f \\
& \text { if } e_{p}>e_{\max } \text { then } \\
& t_{v} \leftarrow t_{v}+T_{s} \\
& \text { else if } e_{p}<-e_{\max } \text { then } \\
& t_{v} \leftarrow t_{v}-T_{s} \\
& \text { end if } \\
& t_{v} \leftarrow t_{v} \cdot f_{\text {old }} / f \\
& \text { if } t_{v}>T \text { then } \\
& t_{v} \leftarrow t_{v}-T \\
& \text { else if } t_{v}<0 \text { then } \\
& t_{v} \leftarrow t_{v}+T \\
& \text { end if } \\
& \mathbf{V} \leftarrow \mathbf{T} \cdot \mathbf{f}\left(t_{v}\right) \\
& \mathbf{D} \leftarrow V_{s}^{-1} \cdot \mathbf{V} \\
& \text { end while } \\
& \text { : end procedure }
\end{aligned}
$$

The algorithm was discussed more in detail in previous publications. Here, it is given for the sake of completeness of this paper. The difference of the algorithm presented with 
respect to the previously published version is that input to the controller is now position error $e_{p}$, while previously inputs were the reference position and measured position. In the algorithm, statements in lines 8-14 calculate the new frequency of the vector $\mathbf{f}$ components, based on the absolute value of the position error. This frequency modulation ensures no overshoot high precision positioning. Based on the position error, the virtual time $t_{v}$ is increased or decreased for the length of the sampling interval $T_{s}$. This change is not done if the error is in the range $\left[-e_{\max }, e_{\max }\right]$. The virtual time is then scaled, in order to ensure that driving voltages do not change considerably between two consecutive sampling intervals. The last steps in the algorithm calculate the vector of PWM duties to be sent to four PWM channels which control four phases of the power driver [27]. The power driver, supplied with voltage $V_{s}$ generates the driving voltages for the motor. The driver is discussed in detail in [27].

The structure of the proposed control system is shown in Fig. 5. Based on the reference force $F^{\text {ref }}$ and measured force $F$, the force tracking error $e_{F}$ is calculated. The measured force is the filtered raw signal $F_{\text {raw }}$ obtained as the output from the force measurement setup, and the filtering was done due to the noisy nature of the $F_{\text {raw }}$ signal. The used filter was a low-pass filter with cut-off frequency of $100 \mathrm{~Hz}$. According to (6), the force error is transformed to the position error $e_{p}$. The position control algorithm tends to bring the position error close to zero, which will consequently bring the measured force close to the reference force. It has to be noted that parameter $k_{e}$ in Fig. 5 does not have to be necessarily equal to the spring constant of the environment, as one can be changing it as a control parameter in the force control loop. In this way, it can be considered as proportional gain in the control loop. If the spring constant of the environment could be identified correctly, reference position $x^{r e f}$ could be generated directly from the reference force, but closed loop position control would still be needed.

\section{EXPERIMENTAL RESUlTS}

A series of experiments was performed to validate the presented force control scheme. Initially, values of the experimental parameters used were as follows: $b=34 \mathrm{~V}, d=50$ $\mathrm{V}, c_{\text {min }}=18.5 \mathrm{~V}, T_{s}=50 \mu \mathrm{s}, f_{\text {max }}=500 \mathrm{~Hz}, f_{\text {min }}=5 \mathrm{~Hz}$, $e_{\text {up }}=5 \mu \mathrm{m}, e_{\text {down }}=0.25 \mu \mathrm{m}, e_{\text {max }}=10 \mathrm{~nm}, k_{e}=3 \cdot 10^{5} \mathrm{~N} / \mathrm{m}$. The control algorithm was executed on the dSPACE's DS1103 control platform.

The results of the first experiment are given in Fig. 6. The reference force was given as a step of $3 \mathrm{~N}$. The force response is satisfactory, as reference is reached in less than $0.2 \mathrm{~s}$, with no overshoot. The force tracking error in steady state is less than $0.02 \mathrm{~N}$.

In order to test controller's operation in both directions and its repeatability, an experiment was performed with a pulse reference of $4 \mathrm{~N}$. The recorded results are shown in 7. The controller's performance is again satisfactory, as reference is reached within $0.3 \mathrm{~s}$ in both directions, and steady state error is less than $0.02 \mathrm{~N}$, as in the previous experiment.
It has been claimed that $y$-direction interaction force has to be large enough so the motor can overcome load force acting in the $x$-direction. The $y$-direction interaction force ensures no relative motion between the legs and the rod. This force is being controlled by the parameter $d$, which sets the amplitude of the vector $\mathbf{f}$ components, $f_{1 p 1}$ and $f_{1 p 2}$. In order to confirm this, an experiment in which $d$ was changing during the experiment was made, and it was done after the reference force is reached. The results are depicted in Fig. 8. One can see that motor lost its ability to keep the force at 5 $\mathrm{N}$ after $d$ is decreased to approximately $28 \mathrm{~V}$. It then slowly started to move towards the reference force, but it was able to keep the force in vicinity of the reference at the end of experiment, when $d$ reached $37 \mathrm{~V}$. This means that successful force control can be done only if $y$-direction interaction force is at appropriate level. In all depicted responses, delay of the force convergence is due to the frequency modulation of the vector $\mathbf{f}$ components.

\section{CONClusion AND FUture WORK}

In this paper, a force control algorithm for a walking piezoelectric motor was presented. The algorithm is very simple, but capable of providing effective force control. The motor is driven according to the coordinate transformation presented in our previous works. Using the transformation, one can synthesize driving voltages for the motor according to a desired motion of the motor legs in the plane of motion. Therefore, it is possible to create conditions for no relative motion between the legs and the rod, and then control the $x$ direction force imposed by the rod on its environment. The control of the $x$-direction force is based on the previously presented position control algorithm named virtual time control.

The future work will be concerned with specific applications of the Piezo LEGS motor, which demand high precision nanometric positioning and force control.

\section{ACKNOWLEDGMENT}

The authors would like to thank TÜBİTAK Project 114M578 for providing financial support for this work.

\section{REFERENCES}

[1] S. Yang, R. A. MacLachlan, and C. N. Riviere, "Manipulator design and operation of a six-degree-of-freedom handheld tremor-canceling microsurgical instrument," IEEE/ASME Trans. Mechatronics, vol. 20, no. 2, pp. 761-772, Apr. 2015.

[2] F. Sergi, A. C. Erwin, and M. K. O'Malley, "Interaction control capabilities of an mr-compatible compliant actuator for wrist sensorimotor protocols during fmri," IEEE/ASME Trans. Mechatronics, vol. 20, no. 6, pp. 2678-2690, Dec. 2015

[3] Y. L. Zhang, Y. Zhang, C. Ru, B. K. Chen, and Y. Sun, "A load-lockcompatible nanomanipulation system for scanning electron microscope," IEEE/ASME Trans. Mechatronics, vol. 18, no. 1, pp. 230-237, Feb. 2013.

[4] H. Su, W. Shang, G. Cole, G. Li, K. Harrington, A. Camilo, J. Tokuda, C. M. Tempany, N. Hata, and G. S. Fischer, "Piezoelectrically actuated robotic system for mri-guided prostate percutaneous therapy," IEEE/ASME Trans. Mechatronics, vol. 20, no. 4, pp. 1920-1932, Aug. 2015.

[5] J.-W. Wu, K.-C. Huang, M.-L. Chiang, M.-Y. Chen, and L.-C. Fu, "Modeling and controller design of a precision hybrid scanner for application in large measurement-range atomic force microscopy," IEEE Trans. Ind. Electron., vol. 61, no. 7, pp. 3704-3712, Jul. 2014. 


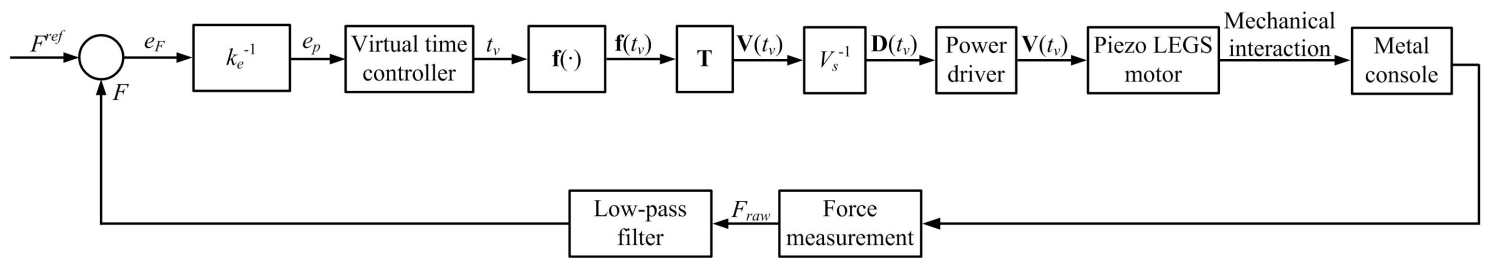

Fig. 5: Control system structure.

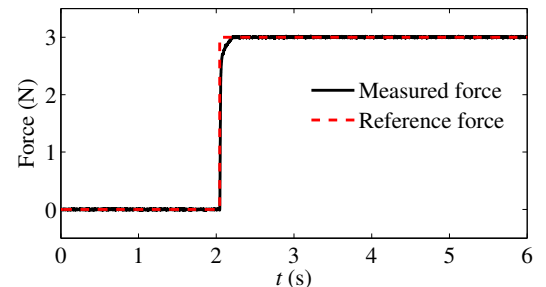

Fig. 6: Force response for $3 \mathrm{~N}$ step reference.

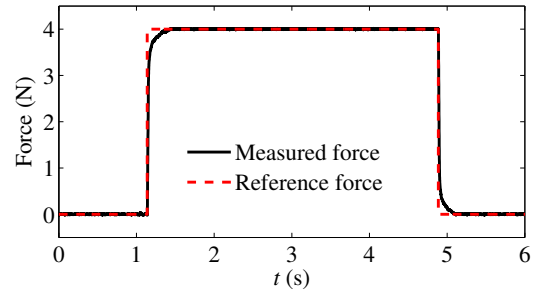

Fig. 7: Force response for $4 \mathrm{~N}$ pulse reference.
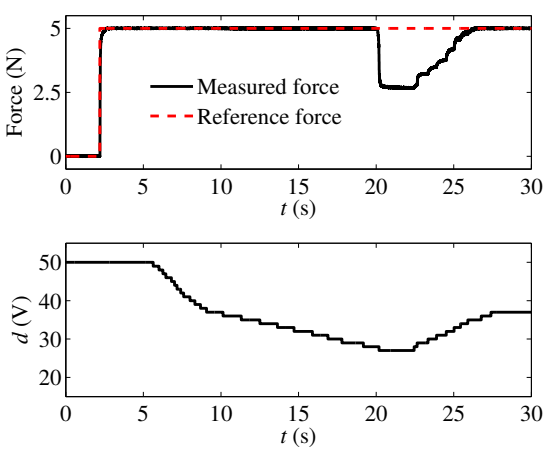

Fig. 8: Force response for $5 \mathrm{~N}$ step reference and variable parameter $d$.

[6] L. Clark, B. Shirinzadeh, U. Bhagat, J. Smith, and Y. Zhong, "Development and control of a two dof linear-angular precision positioning stage," Mechatronics, vol. 32, pp. 34-43, Dec. 2015.

[7] G.-Y. Gu, L.-M. Zhu, C.-Y. Su, and H. Ding, "Motion control of piezoelectric positioning stages: modeling, controller design, and experimental evaluation," IEEE/ASME Trans. Mechatronics, vol. 18, no. 5, pp. 14591471, Oct. 2013.

[8] G.-Y. Gu, L.-M. Zhu, C.-Y. Su, H. Ding, and S. Fatikow, "Modeling and control of piezo-actuated nanopositioning stages: a survey," IEEE Trans. Autom. Sci. Eng., vol. 13, no. 1, pp. 313-332, Jan. 2016.

[9] C.-X. Li, G.-Y. Gu, M.-J. Yang, and L.-M. Zhu, "Positive position feedback based high-speed tracking control of piezo-actuated nanopositioning stages," in Intelligent Robotics and Applications. Springer,
2015, pp. 689-700.

[10] M. Butcher, A. Giustiniani, R. Losito, and A. Masi, "Controller design and verification for a rotational piezo-based actuator for accurate positioning applications in noisy environments," in IECON 2015-41st Annual Conference of the IEEE Industrial Electronics Society, 2015, pp. $003887-003892$

[11] C.-X. Li, G.-Y. Gu, M.-J. Yang, and L.-M. Zhu, "High-speed tracking of a nanopositioning stage using modified repetitive control," IEEE Trans. Autom. Sci. Eng., DOI: 10.1109/TASE.2015.2428437, Publication date: 22 May 2015.

[12] A. A. Eielsen, J. T. Gravdahl, and K. K. Leang, "Low-order continuoustime robust repetitive control: Application in nanopositioning," Mechatronics, vol. 30, pp. 231-243, Sep. 2015.

[13] G.-Y. Gu, C.-X. Li, L.-M. Zhu, and C.-Y. Su, "Modeling and identification of piezoelectric-actuated stages cascading hysteresis nonlinearity with linear dynamics," IEEE/ASME Trans. Mechatronics, DOI: 10.1109/TMECH.2015.2465868, Publication date: 07 August 2015.

[14] S. Johansson, M. Bexell, and P. O. Lithell, "Fine walking actuator," U.S. Patent 6337 532, Jan. 8, 2002.

[15] — "Switched actuator control," U.S. Patent 6459 190, Oct. 1, 2002

[16] S. Johansson, M. Bexell, and A. Jansson, "Fine control of electromechanical motors," U.S. Patent 6798 117, Sep. 28, 2004.

[17] R. J. E. Merry, N. C. T. de Kleijn, M. J. G. van de Molengraft, and M. Steinbuch, "Using a walking piezo actuator to drive and control a high-precision stage," IEEE/ASME Trans. Mechatronics, vol. 14, no. 1, pp. 21-31, Feb. 2009.

[18] R. J. E. Merry, D. J. Kessels, W. P. M. H. Heemels, M. J. G. van de Molengraft, and M. Steinbuch, "Delay-varying repetitive control with application to a walking piezo actuator," Automatica, vol. 47, no. 8, pp. 1737-1743, Aug. 2011

[19] E. Golubovic, T. Uzunovic, Z. Zhakypov, and A. Sabanovic, "Adaptive control of piezoelectric walker actuator," in Proc. IEEE Int. Conf. Mechatronics, 2013, pp. 132-137.

[20] R. J. E. Merry, J. L. Holierhoek, M. J. G. van de Molengraft, and M. Steinbuch, "Gain scheduling control of a walking piezo actuator," IEEE/ASME Trans. Mechatronics, vol. 19, no. 3, pp. 954-962, Jun. 2014

[21] F. Szufnarowski, A. Gollin, and A. Schneider, "Insect-inspired drive strategy substantially improves the performance of a piezo motor," Sensors and Actuators A: Physical, vol. 219, pp. 13-23, Nov. 2014.

[22] T. Uzunovic, E. Golubovic, and A. Sabanovic, "FPGA based control of a walking piezo motor," in Proc. 13th Int. Workshop Adv. Motion Control, 2014, pp. 138-143.

[23] T. Uzunovic, E. Golubovic, D. Kebude, and A. Sabanovic, "Control system for high precision positioning applications based on piezo motors," in Proc. 2015 IEEE Int. Conf. Mechatronics, 2015, pp. 116121.

[24] F. Szufnarowski and A. Schneider, "Force control of a piezoelectric actuator based on a statistical system model and dynamic compensation," Mechanism and Machine Theory, vol. 46, no. 10, pp. 1507-1521, Oct. 2011.

[25] - "Compliant piezo-flexdrives for muscle-like, antagonistic actuation of robot joints," in Proc. 3rd IEEE Int. Conf. Biomed. Robot. Biomechatronics, 2010, pp. 381-388.

[26] T. Uzunovic, E. Golubovic, and A. Sabanovic, "Piezo legs driving principle based on coordinate transformation," IEEE/ASME Trans. Mechatronics, vol. 20, no. 3, pp. 1395-1405, Jun. 2015.

[27] E. Golubovic, Z. Zhakypov, T. Uzunovic, and A. Sabanovic, "Piezoelectric motor driver: design and evaluation," in Proc. 39th Annu. Conf. IEEE Ind. Electron. Soc., 2013, pp. 3964-3969. 